\title{
Osteoblasts from osteoarthritis patients show enhanced susceptibility to Ross River virus infection associated with delayed type I interferon responses
}

Weiqiang Chen ${ }^{1+}$, Suan-Sin Foo ${ }^{1+}$, Rachel W Li ${ }^{2}$, Paul N Smith ${ }^{3}$ and Suresh Mahalingam ${ }^{1 *}$

\begin{abstract}
Background: Arthritogenic alphaviruses such as Ross River virus (RRV) and chikungunya virus (CHIKV) have caused widespread outbreaks of chronic polyarthritis. The inflammatory responses in alphavirus-induced arthritis and osteoarthritis $(\mathrm{OA})$ share many similar features, which suggests the possibility of exacerbated alphavirus-induced bone pathology in individuals with pre-existing OA. Here, we investigated the susceptibility of osteoblasts (OBs) from OA patients to RRV infection and dissected the immune mechanisms elicited from infection.

Methods: Primary hOBs obtained from trabecular bone of healthy donors and OA patients were infected with RRV. Infectivity and viral replication were determined using flow cytometry and plaque assay, respectively. Real-time PCR was performed to determine expression kinetics of type I interferon (IFN)-related immune mediators and osteotropic factors.

Results: OA hOBs showed enhanced RRV infectivity and replication during infection, which was associated with delayed induction of IFN- $\beta$ and RIG-I expression. Enhanced susceptibility of OA hOBs to RRV was associated with a more pronounced increase in RANKL/OPG ratio and expression of osteotropic factors (IL-6, IL-1 $\beta$, TNF-a and CCL2) in comparison to RRV-infected healthy hOBs.

Conclusions: Delayed activation of type I IFN-signalling pathway may have contributed to enhanced susceptibility to RRV infection in hOBs from OA patients. RRV-induced increases in RANKL/OPG ratio and expression of osteotropic factors that favour bone resorption, which may be exacerbated during osteoarthritis. This study provides the novel insight that osteoarthritis may be a risk factor for exacerbated arthritogenic alphaviral infection.
\end{abstract}

Keywords: Ross River virus, Human osteoblasts, RANKL/OPG ratio, Osteotropic factors

\section{Background}

Ongoing global warming and increased rainfall variability globally have provided extensive breeding grounds for arthropod vectors. Infection rates of arthropod-borne alphaviruses such as Ross River virus (RRV) and chikungunya virus (CHIKV) are currently rising in different geographic regions of the world [1]. Debilitating polyarthralgia/polyarthritis is the clinical hallmark of RRV and CHIKV infection, which typically affects ankle joints, knees and peripheral joints [2-4]. The onset of RRV disease (RRVD) and chikungunya fever (CHIKF) can be

\footnotetext{
* Correspondence: s.mahalingam@griffith.edu.au

${ }^{\dagger}$ Equal contributors

${ }^{1}$ Emerging Viruses and Inflammation Research Group, Institute for Glycomics, Griffith University, Gold Coast Campus, QLD 4222, Australia

Full list of author information is available at the end of the article
}

sudden and debilitating, with the level of disability comparable to other forms of arthritis such as osteoarthritis (OA) or chronic rheumatoid arthritis (RA) [5].

Prolonged manifestations of chronic RRVD and CHIKF, including persistent polyarthritis and/or polyarthralgias, occur in approximately $40 \%$ of infected individuals [6]. Pro-inflammatory immune mediators, including interleukin (IL)-6, IL-1, tumor necrosis factor- $\alpha$ (TNF- $\alpha$ ) and monocyte chemotactic protein-1 (MCP-1; or CCL2) have been shown to contribute to the progression of alphaviral disease [7-9]. Interestingly, these pro-inflammatory cytokines are also known as osteotropic factors that modulate the bone remodelling system.

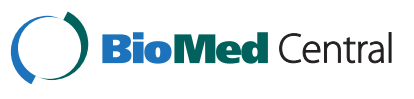

(c) 2014 Chen et al.; licensee BioMed Central Ltd. This is an Open Access article distributed under the terms of the Creative Commons Attribution License (http://creativecommons.org/licenses/by/4.0), which permits unrestricted use, distribution, and reproduction in any medium, provided the original work is properly credited. The Creative Commons Public Domain Dedication waiver (http://creativecommons.org/publicdomain/zero/1.0/) applies to the data made available in this article, unless otherwise stated. 
The human bone remodelling system requires the coordinated action of bone-resorbing osteoclasts (OCs) and bone-forming osteoblasts (OBs), which are together termed the basic multicellular unit (BMU), to maintain bone homeostasis. OBs are involved in bone formation and mineralisation and orchestrate cell signalling pathways required for local control of OC differentiation [10]. In most arthritic conditions, OCs have been identified as the principal cells responsible for bone erosions [11]. Enhanced production of key OC differentiation factors such as receptor activator of nuclear factor (NF) $-\kappa \beta$ ligand (RANKL), IL- 6 , IL- 1 and TNF- $\alpha$ by OBs can stimulate osteoclastogenesis $[10,12]$. To maintain bone homeostasis, osteoclastogenesis is kept in check by OB-secreted osteoprotegerin (OPG), which inhibits OC differentiation by acting as a soluble decoy receptor for RANKL. Hence, an increase in RANKL/OPG ratio is associated with increased $\mathrm{OC}$ activity, which may result in osteolytic pathologies [13-15].

Clinical evidence of bony erosions has been reported in the joints of CHIKV-infected patients [16], providing evidence that alphavirus-induced disease can result in erosive arthritis. Recently, we reported pathologic bone loss during acute RRV infection in an acute RRVD mouse model, which is consistent with the increased tartrate-resistant acid phosphatase 5b (TRAP5b) level and RANKL/OPG ratio in serum of RRV patients. RRV infection of primary human osteoblasts (hOBs) also leads to an increased RANKL/OPG ratio and robust production of pro-inflammatory cytokines IL-6 and CCL2 [17].

$\mathrm{OA}$, also known as degenerative joint arthritis, is the most common form of arthritis. It is characterized by degradation of cartilage and inflammation of the synovium and affects millions of people worldwide, with an estimate of over 1.4 million people affected in Australia alone [18]. Pro-inflammatory cytokines such as IL-6, IL1 , TNF- $\alpha$ and CCL2 are involved in OA severity: this cytokine profile has a striking resemblance to inflammatory responses elicited in alphavirus-induced arthritis [19]. The shared cytokine profile between alphavirusinduced arthritis and OA highlights a potential for exacerbation of alphavirus-induced bone loss in individuals with pre-existing OA $[3,20,21]$.

In this study, we investigated the potential of underlying $\mathrm{OA}$ to influence alphavirus infection. RRV infection of OA hOBs led to enhanced viral infectivity and replication, accompanied by delayed type I IFN responses. RRV-induced pro-inflammatory immune responses were increased in OA hOBs. The imbalance in the RANKL/ OPG axis that results from RRV infection was further perturbed in hOBs from OA patients, suggesting OA as a predisposing risk factor for alphavirus-induced bone pathology.

\section{Results}

Enhanced RRV infectivity in primary hOBs of OA patients, modulated through delayed type IFN signalling pathway

To investigate if underlying OA has any effect on viral replication during RRV infection, primary hOBs cultured from trabecular bones of healthy donors and OA patients were used. The cultured primary hOBs were phenotypically characterized using osteocalcin, an OB marker, prior to RRV infection (Figure 1A). To determine the infectivity of these primary hOB cultures, they were infected with RRV-EGFP at a series of multiplicity of infection (MOI) from 1 to 10 . Infectivity of both healthy and OA hOBs was demonstrated in a dose-dependent manner (Figure 1B). The kinetics of RRV replication in the primary hOBs was further determined through a $96 \mathrm{~h}$ time-course infection, with cells and supernatant harvested at 24, 48 and 96 hours post-infection (hpi) for FACS analysis and plaque assays. Peak RRV replication was observed at 24 hpi for both healthy and OA hOBs, with a subsequent decline over the next $72 \mathrm{~h}$. Interestingly, FACS analysis of the harvested cells revealed significantly enhanced RRV infectivity in OA hOBs at 24, 48 and 96 hpi (Figure 1C). Consistent with the enhanced infectivity in OA hOBs, higher levels of virus were also recovered from the supernatant of infected OA hOBs at 24 and $48 \mathrm{hpi}$, compared to healthy hOBs (Figure 1D).

The type I IFN signalling pathway serves as the first line of host defence against pathogen invasion. We sought to determine the involvement of type I IFN in the contribution of enhanced RRV infectivity and replication in OA hOBs. Infected primary hOBs were harvested at 24, 48 and 96 hpi for determination of IFN- $\beta$ and RIG-I mRNA expression (Figure 2). At the peak viral replication of $24 \mathrm{hpi}$, RRV-infected OA hOBs had significantly lower transcriptional induction of both IFN- $\beta$ and RIG-I compared to RRV-infected healthy hOBs. The induction of IFN- $\beta$ and RIG-I in RRV-infected OA hOBs gradually overtook that of RRV-infected healthy hOBs at 48 and 96 hpi, respectively. Together, these data suggest that enhanced susceptibility and viral replication in OA hOBs may be modulated through delayed induction of type I IFN.

\section{Underlying OA further perturbs bone homeostasis after RRV infection}

Alphavirus-induced arthritis and OA are inflammatory arthritides that have been associated with bone disorders $[17,22]$. Recently, we demonstrated that RRV infection can elicit bone loss through perturbed OB function. Therefore, we investigated the effect of RRV infection on bone regulatory function of OA hOBs by measuring RANKL and OPG, which are the fundamental elements of bone homeostasis. Briefly, primary hOB cultures were infected at MOI 1 and harvested at 24, 48 and 96 hpi for determination of RANKL and OPG transcript expression. RANKL expression in OA hOBs was significantly higher at all time points 


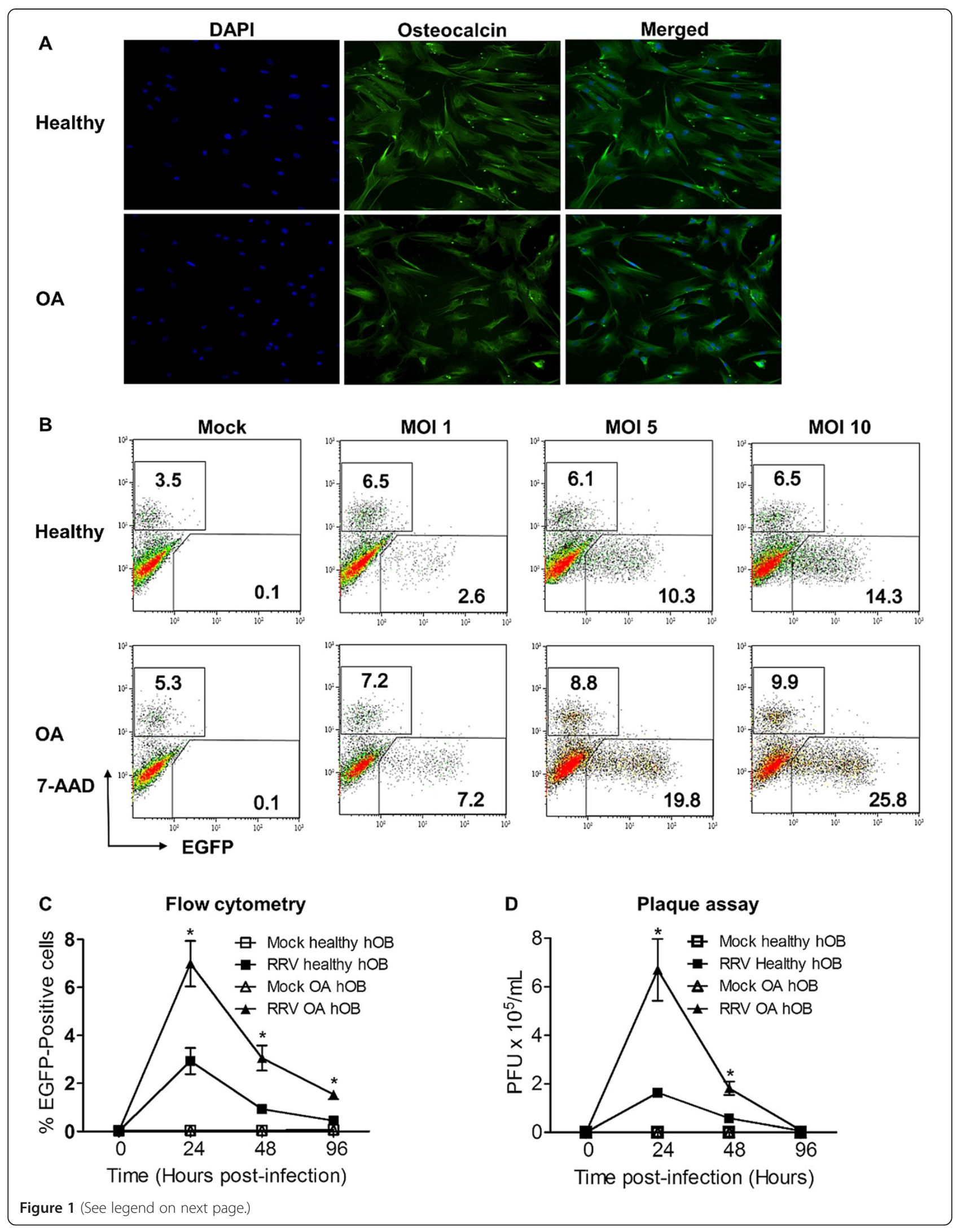


(See figure on previous page.)

Figure 1 Enhanced RRV replication in primary hOBs of OA patients. (A) Phenotypic characterization of primary hOBs. Primary hOBs were fixed and stained for osteocalcin (green) and nuclei (blue), and visualized by confocal microscopy. Magnification, $\times 20$. Images are representative of 2 independent experiments. Primary hOBs were infected with RRV-EGFP at MOI 1 and cells and supernatant were harvested at different time points. (B) Dose-dependent infection of hOBs. Cell harvested at 24 hpi were fixed, stained for 7-AAD, and subjected to FACS analysis. FACS scatter plots are representative of the data obtained for 3 independent experiments. (C) Quantification of percentage EGFP ${ }^{+}$cells after infection. (D) Supernatant was harvested and RRV titre was determined by plaque assay on Vero cells. Data $(n=4)$ are presented as mean \pm SEM. ${ }^{*} P<0.05$, two-way ANOVA, Bonferroni post-test.

than in healthy hOBs after RRV infection. A drastic increase in RANKL expression of $\sim 20$-fold between 24 to 96 hpi was observed in RRV-infected OA hOBs, compared to $\sim 5$-fold increase for RRV-infected healthy hOBs. No significant difference in RANKL expression was observed between mock-infected healthy and OA hOBs (Figure 3A). Higher levels of OPG expression were detected in mockinfected OA hOBs compared to mock- or RRV-infected healthy hOBs. After RRV infection, OA hOBs demonstrated higher expression of OPG compared to healthy infected hOBs at all tested time points. No significant changes in OPG expression were observed between mockor RRV-infected healthy hOBs at all tested time points. However, at 48 and $96 \mathrm{hpi}$, OPG levels were significantly lower in the RRV-infected OA hOBs compared to mockinfected OA hOBs (Figure 3B). Thus, RRV infection resulted in a markedly elevated RANKL/OPG ratio in both healthy and OA hOBs compared to mock-infected groups. Interestingly, OA hOBs exhibited a significantly higher RANKL/OPG ratio than healthy hOBs after RRV infection at 48 and 96 hpi (Figure 3C), suggesting that underlying OA may exacerbate RRV-induced bone pathology by further increasing the RANKL/OPG ratio.

\section{Increased expression of osteotropic factors in primary} hOBs of OA patients after RRV infection

Pro-inflammatory mediators such as IL-6, IL- $1 \beta$, TNF- $\alpha$ and CCL2 contribute to alphaviral disease pathogenesis.

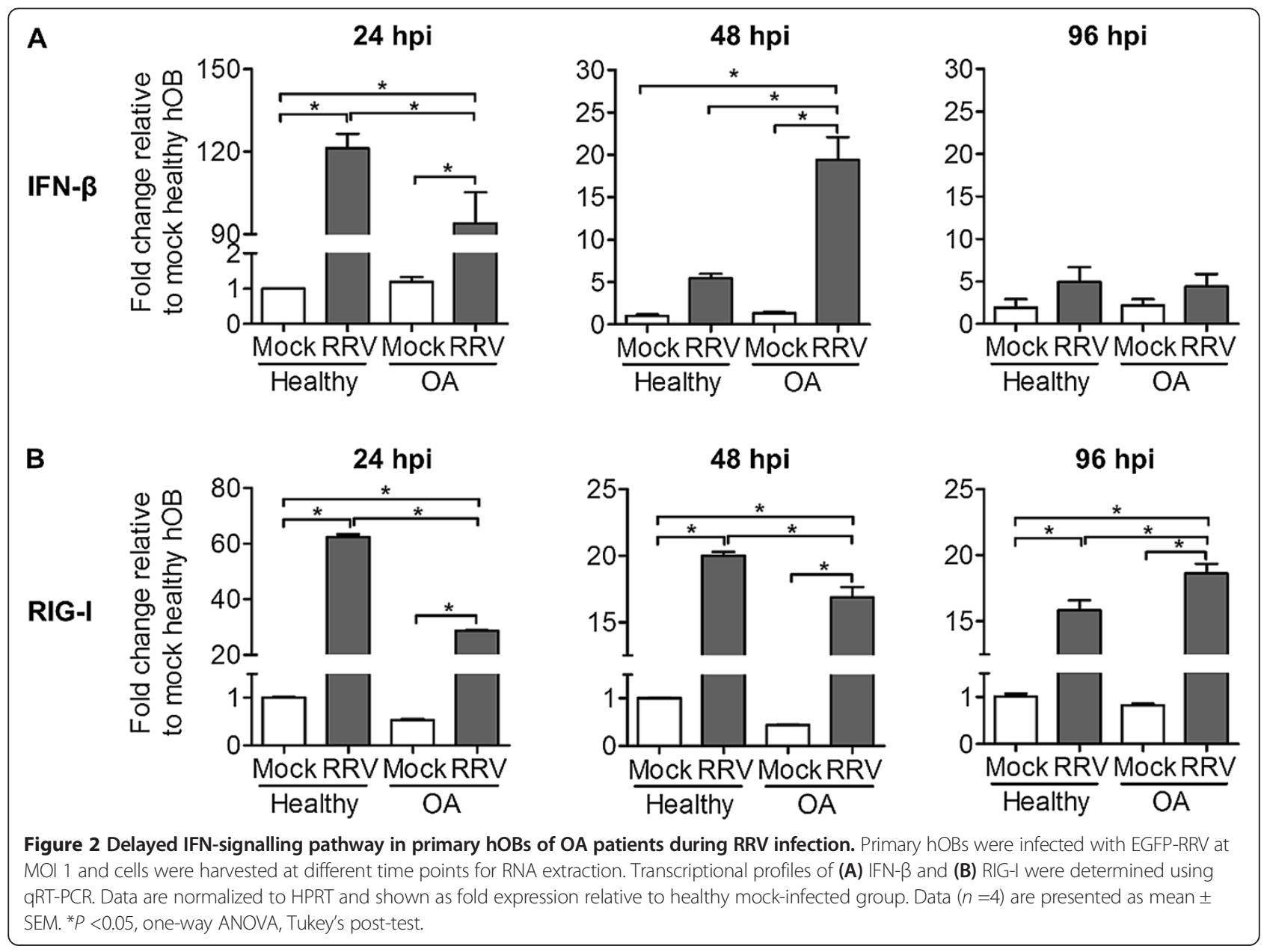




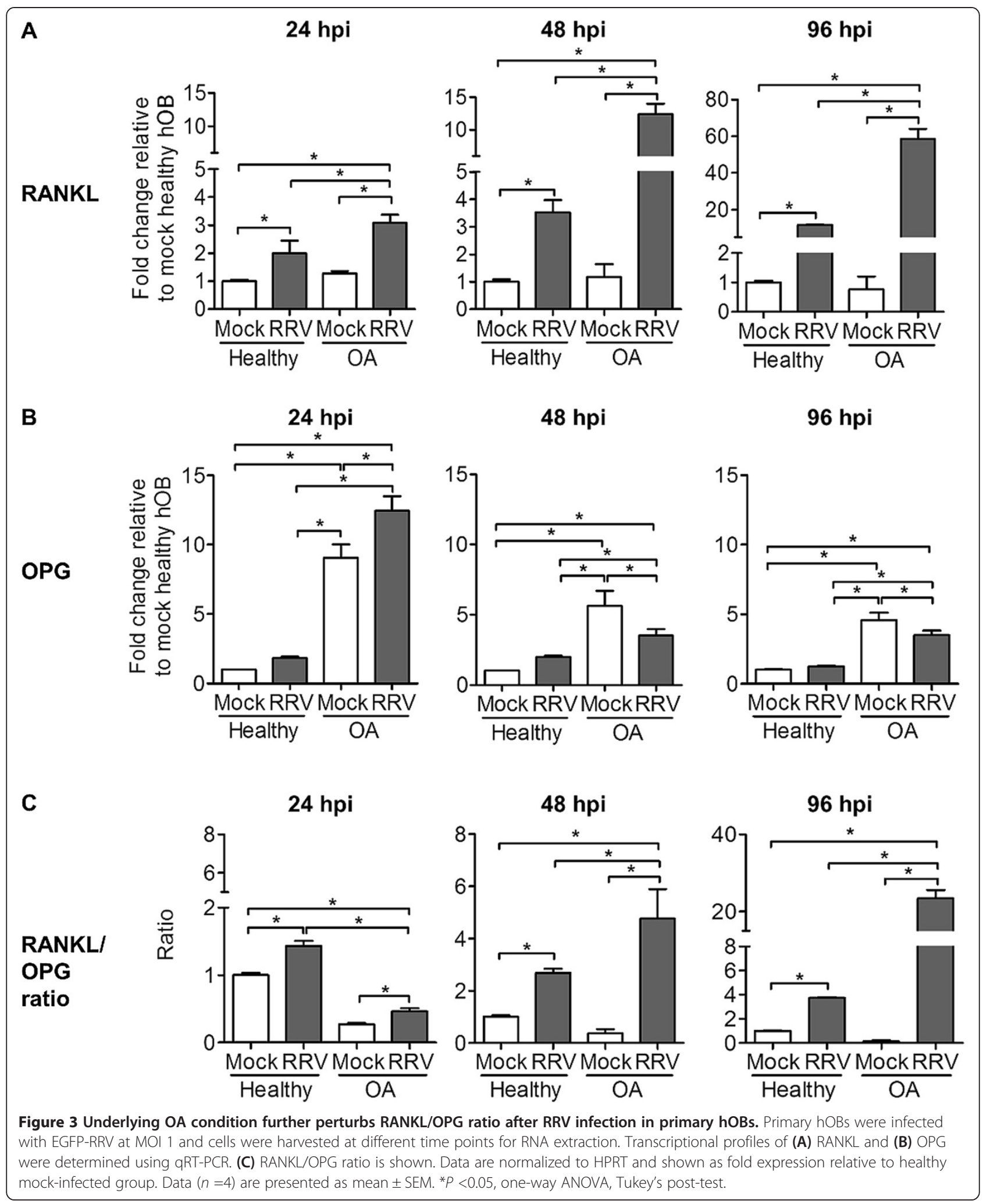

Interestingly, these immune mediators also function as osteotropic factors, with a pivotal role in modulating bone remodelling $[23,24]$. Transcriptional analysis of these osteotropic factors was performed to assess their expression kinetics. IL-6 expression was up-regulated at all tested time points after RRV infection in both 
healthy and OA hOBs. At 24 and 48 hpi, RRV infection of OA hOBs elicited consistently lower levels of IL-6 transcription compared to healthy hOBs, but its expression was increased at 96 hpi (Figure 4A). Early induction of IL-1 $\beta$ expression was observed in both healthy and OA hOBs after RRV infection at $24 \mathrm{hpi}$, which peaked at $48 \mathrm{hpi}$, with $\sim 30$-fold increase for RRV-infected healthy hOBs and $\sim 90$-fold increase for RRV-infected OA hOBs. At $96 \mathrm{hpi}$, the RRV-induced expression of IL- $1 \beta$ in healthy hOBs declined, while transcription continued to increase dramatically to $~ 600$-fold change in RRV-infected OA hOBs (Figure 4B). Elevated expression of TNF- $\alpha$ was detected at 24 hpi in RRV-infected healthy and OA hOBs, but to a lesser extent for the OA group. The induction of TNF- $\alpha$ declined rapidly at $48 \mathrm{hpi}$ and reverted to basal level by 96 hpi (Figure 4C). CCL2 expression was highly up-regulated in healthy hOBs after RRV infection at 24 hpi and decreased gradually over the next $72 \mathrm{~h}$. In contrast, RRV-induced CCL2 expression in OA hOBs only commenced at 96 hpi (Figure 4D). Collectively, the elevated expression of these pro-inflammatory/osteotropic factors at later stages of infection may stimulate RANKLmediated osteoclastogenesis.

\section{Discussion}

Alphavirus-infected patients suffering from pre-existing joint disorders such as OA have been reported to be at possible increased risk of severe joint arthralgia and delayed remission $[3,16,20,25,26]$. However, the mechanism behind prolonged and exacerbated disease in patients with underlying arthritis has not been elucidated. Our previous findings highlighted that alphaviruses possess tropism for murine bones and that OB-lineage cells have potential roles in the pathogenesis of alphaviral arthritis $[17,27,28]$. This study was undertaken to investigate the susceptibility and response of primary hOB from OA patients to arthritogenic RRV infection and to analyse if infection can exacerbate hOB osteoclastogenic potential.

Primary hOBs from OA patients were significantly more susceptible to RRV infection and the virus replicated at higher levels compared to hOBs from healthy donors. Alphaviruses are susceptible to type I IFNs, which was elicited in response to RNA viruses by the cytoplasmic RNA sensor RIG-I [29-32]. A possible explanation for the enhanced infection of OA hOBs was the lower expression of RIG-I and IFN- $\beta$ compared to healthy cells at peak infection (24 hpi), suggesting a delayed IFN-signalling pathway in OA hOBs.

Bone cells such as OB play a pivotal role in bone remodelling, with primary role in bone formation and regulation of $\mathrm{OC}$ bone resorption through the OPG/ RANKL/RANK system [33,34]. Cells of OB lineage express both RANKL and OPG, which are key osteotropic factors in regulating bone remodelling; a higher
RANKL/OPG ratio favours osteoclastogenesis [13-15]. At basal levels, we found a lower RANKL/OPG ratio in OA hOBs than healthy hOBs. This is in agreement with previous studies showing that $\mathrm{OA}$ patients have a lower RANKL/OPG ratio, suggesting reduced osteoclastic activity $[35,36]$. In contrast to the lower RANKL/OPG ratio in OA hOBs, the ratio in these cells was significantly increased after $96 \mathrm{~h}$ RRV infection compared to healthy infected cells, highlighting the potential of OA predisposed to exacerbated alphavirus-induced bone pathology.

Pro-inflammatory/osteotropic factors are crucial in both bone remodelling and in the pathogenesis of alphavirus infection. For example, IL-6 is highly expressed in affected joints during alphavirus disease [37] and is proposed to be an index of CHIKV disease severity $[8,38]$. Recently, we showed that IL-6, IL-1 $\beta$, TNF- $\alpha$ and CCL2 are highly expressed in RRV-infected hOBs of healthy donors and that these infected cells are crucial in mediating RRV-induced bone loss through the up-regulation of RANKL/OPG ratio [17]. Similarly, we observed upregulation of IL-6, IL-1 $\beta$ and TNF- $\alpha$ in OA hOBs at peak infection (24 hpi) in our study. Although RRVinduced IL- 6 , TNF- $\alpha$ and CCL2 were lower in the OA hOBs compared to infected controls during early infection, the expression of these osteotropic factors in $\mathrm{OA}$ hOBs, with the exception of TNF- $\alpha$, was significantly elevated at 96 hpi. Hence, the increased expression of IL$6, I L-1 \beta$ and CCL2 at later stages of infection may act to promote osteoclastogenesis. This is supported by the high RANKL/OPG ratio, a well-established index for osteoclastogenesis, in $\mathrm{OA}$ hOB after 96 hpi compared to infected controls.

Our in vitro findings are the first to demonstrate increased susceptibility of primary OA hOBs to RRV infection, which may contribute to disease exacerbation in patients with underlying inflammatory arthritis.

\section{Conclusions}

These exciting results clearly indicate that the response of 'arthritic' osteoblasts to RRV infection differs from normal osteoblasts; they are more susceptible to infection possibly through delayed type I IFNs response and produce higher levels of IL-6, IL-1 $\beta$, CCL2 and TNF- $\alpha$. At later stages of infection, RRV-infected OA hOBs showed significantly increased IL-6, IL- $1 \beta$ and CCL2 expression compared to healthy infected controls. The increase in these pro-inflammatory/osteotropic factors by OA hOBs may act to recruit monocytic OC precursor cells to sites of infection and to enhance the expression of RANKL in RRV-infected hOBs. These delayed pro-inflammatory responses resulted in an increased RANKL/OPG ratio, which can lead to increased osteoclastogenesis. Our novel findings highlight a possible 


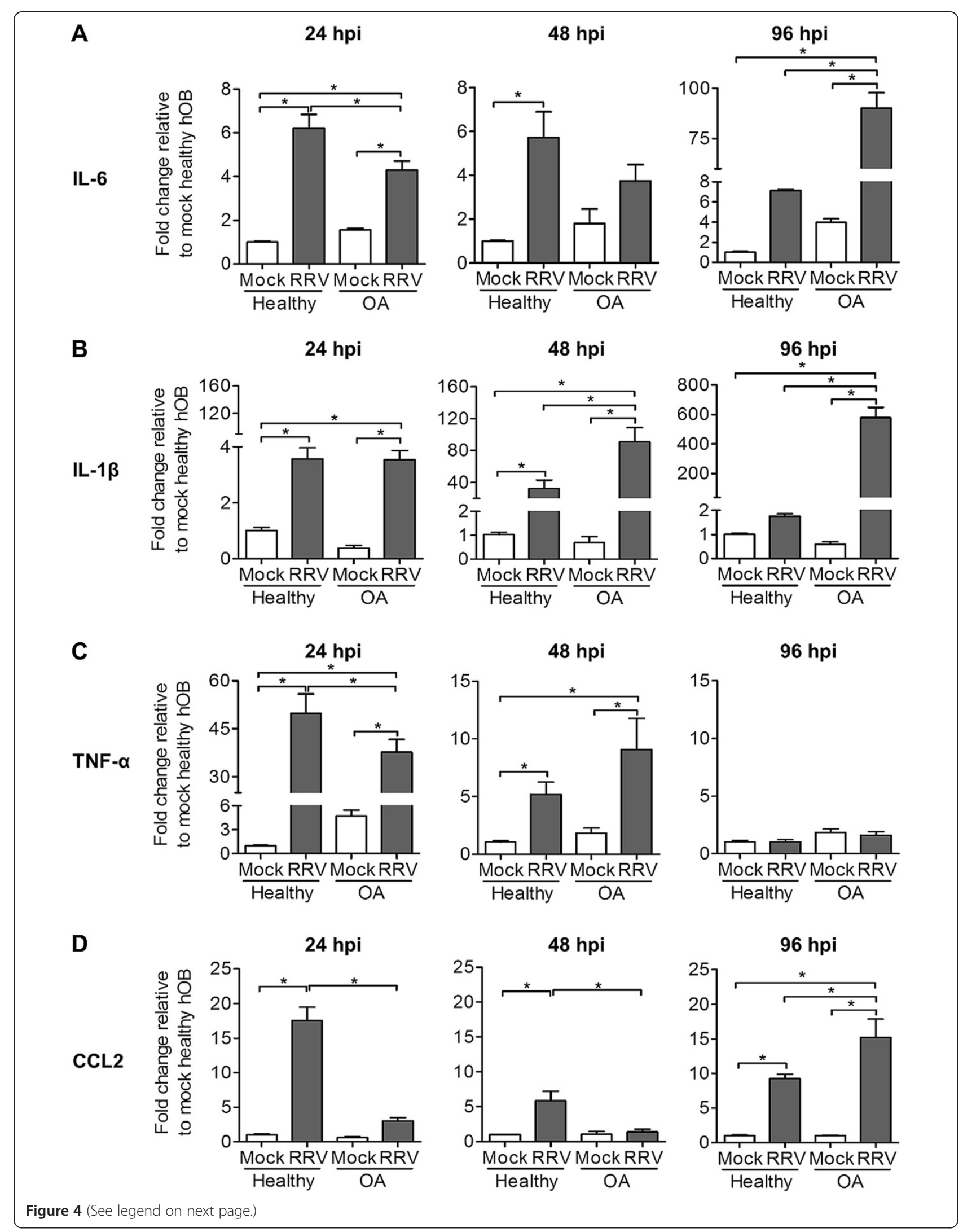


(See figure on previous page.)

Figure 4 Enhanced expression of osteotropic factors in primary hOBs of OA patients after RRV infection. Primary hOBs were infected with EGFP-RRV at MOI 1 and cells were harvested at different time points for RNA extraction. Transcriptional profiles of osteotropic factors (A) IL-6, (B) IL-1 $\beta$, (C) TNF-a and (D) CCL2 were determined using qRT-PCR. Data are normalized to HPRT and shown as fold expression relative to healthy mock-infected group. Data $(n=4)$ are presented as mean \pm SEM. ${ }^{*} P<0.05$, one-way ANOVA, Tukey's post-test.

OB-mediated mechanism to explain exacerbations of joint arthralgia and prolonged disease severity after RRV infection in patients with underlying OA.

\section{Methods}

\section{Viruses}

Stocks of RRV strain T48 (RRV-T48) were generated from the full-length T48 cDNA clone [39]. Stocks of RRV that expressed enhanced green fluorescent protein (RRV-EGFP) were generated by inserting a second RRV $26 \mathrm{~S}$ promoter sequence at the 3' end of the viral genome, followed by the coding sequence for EGFP (kindly provided by Dr Mark Heise, University of North Carolina). All titrations were performed by plaque assay on Vero cells as described previously [40].

\section{Primary cell cultures}

Primary human $\mathrm{OB}(\mathrm{hOB})$ cultures were obtained from trabecular bone specimens of 7 healthy individuals (4 males, 3 females), aged 50-60 years. Healthy hOBs were obtained from individuals undergoing orthopaedic operations for causes unrelated to arthritis and osteoporosis (such as ligament reconstruction or trauma cases). OA trabecular bone specimens were obtained from femur neck of 5 patients ( 3 males, 2 females) undergoing primary or revision surgery. The patients ranged in age from $50-60$ years and were diagnosed with OA according to the World Health Organisation (WHO) criteria, mainly based on clinical presentation, radiographic findings. All human specimens were collected with the permission of the Human Research Ethics Committee of Australian National University (human ethics approval-ETH-9-07-865) and Australian Capital Territory (ACT) Health, together with informed patient consent. The exclusion criteria for recruiting hOB donors was (i) taking immune suppressant/stimulating drugs, cytokine, or IFN therapy in the 3 months before surgery; (ii) treatment with anabolic agents; and (iii) low quality of the bone fragment, socio-economic or ethic characteristics and age not matching with the healthy group, in the opinion of the investigators, could compromise the study. Briefly, the bone fragments were washed with PBS and cultured in $\alpha$-MEM (Sigma-Aldrich) supplemented with 10\% HI-FCS, 1\% antibiotic solution, $100 \mu \mathrm{M}$ ascorbic acid, $20 \mathrm{mM}$ Hepes, and $2 \mathrm{mM} \mathrm{L}$-glutamine at $37^{\circ} \mathrm{C}$ with $5 \% \mathrm{CO}_{2}$. After 2 weeks, outgrowing cells surrounding bone fragments were cultured and designated as "passage 1." The hOBs cultured in our laboratory were able to proliferate in vitro for up to five passages but were used at passage 2 in all experiments.

\section{Phenotypic characterisation of primary human osteoblasts} (hOBs)

To characterise cultured hOB, osteocalcin expression was determined by staining hOB with primary mouse antihuman osteocalcin monoclonal antibodies (R\&D Systems, Minneapolis, USA) and Alexa-Fluor 488 (AF488) goat antimouse IgG (Invitrogen, Melbourne, Victoria, Australia), followed by DAPI staining and visualisation using an FV1000 confocal microscope (Olympus, Australia).

\section{In vitro RRV infection}

Primary hOBs were seeded in 24-well plates at a density of $1 \times 10^{5}$ cells per well overnight. Cells were then infected with RRV-EGFP at a multiplicity of infection (MOI) of 1 . After $1 \mathrm{~h}$, cells were washed twice and cultured in fresh $\alpha$-MEM with $10 \%$ FCS at $37^{\circ} \mathrm{C}$ with $5 \% \mathrm{CO}_{2}$. At appropriate time points, culture medium was collected for plaque assay and cells were harvested for RNA extraction.

\section{Flow Cytometry (FACS)}

Cells were trypsinsed and washed twice in wash buffer (PBS-2\% FCS). Cells were then stained with 7-AAD (7aminoactinomycin) (eBioscience, San Diego, CA) at room temperature for $10 \mathrm{~min}$. Following 7-AAD staining, EGFP expression in RRV-infected cells was analysed using a CyAn ADP flow cytometer and Kaluza software (Beckman Coulter).

\section{Plaque assays}

Vero cells were seeded into 24-well plates at a density of $1 \times$ $10^{5}$ cells per well and cultured to confluence. Serially diluted samples $\left(10^{-1}\right.$ to $\left.10^{-6}\right)$ were added to cell monolayers and incubated for $1 \mathrm{~h}$ at $37^{\circ} \mathrm{C}$ in a $5 \% \mathrm{CO}_{2}$ incubator. Cells were then overlaid with OPTI-MEM (Invitrogen, Melbourne, Victoria, Australia) containing 3\% FCS and 1\% agarose (Sigma Aldrich, Sydney, Australia) and incubated for $48 \mathrm{~h}$ in a $5 \% \mathrm{CO}_{2}$ incubator. The cells were fixed with $1 \%$ formalin and plaques were visualised by staining with $0.1 \%$ crystal violet. Virus titres were expressed as plaque forming units per millilitre (PFU/mL) or PFU per gram of tissue.

\section{Real-time PCR (qRT-PCR)}

RNA was prepared from cell pellets using TRIzol (Invitrogen, Melbourne, Victoria, Australia) according to the manufacturer's instructions. Eluted RNA was stored at $-80^{\circ} \mathrm{C}$. Total RNA was measured by NanoDrop 1000 spectrophotometer (Thermo Scientific). Extracted total RNA (20 ng/ $\mu \mathrm{L})$ was reverse-transcribed using an oligo(dT) 
primer and reverse transcriptase (Sigma Aldrich, Sydney, Australia), according to the manufacturer's instructions. $\mathrm{SYBR}^{\circ}$ Green Real-time PCR was performed using $50 \mathrm{ng}$ of template cDNA on a CFX96 Touch ${ }^{\text {tw }}$ Real-Time PCR System in 96-well plates, using QuantiTect Primer Assay kits (Qiagen, Hilden, Germany) with the following conditions: (i) PCR initial activation step: $95^{\circ} \mathrm{C}$ for $15 \mathrm{~min}, 1$ cycle and (ii) 3-step cycling: $94^{\circ} \mathrm{C}$ for $15 \mathrm{~s}$, follow by $55^{\circ} \mathrm{C}$ for $30 \mathrm{~s}$ and $72^{\circ} \mathrm{C}$ for $30 \mathrm{~s}, 40$ cycles. Amplification specificity was evaluated by a melting curve analysis of PCR products. The fold change in messenger RNA (mRNA) expression relative to mock-infected samples for each gene was calculated with the $\Delta \Delta C t$ method. Briefly, $\Delta \Delta C t=\Delta C t$ (RRV-infected) $\Delta C t$ (Mock-infected) with $\Delta C t=C t$ (gene of interest) $-C t$ (housekeeping gene - HPRT). The fold change for each gene is calculated as $2^{-\Delta \Delta C t}$.

\section{Statistical analysis}

Viral titers and flow cytometry data were statistically evaluated using two-way analysis of variance (ANOVA) with Bonferroni posttest. Real-time PCR data were analyzed using one-way ANOVA with Tukey's posttest. Differences between groups with $\mathrm{P}<0.05$ were considered significant. All data were assessed for normality using the D'Agostino-Pearson normality test before analysis with these parametric tests. All statistical analyses were performed with GraphPad Prism software, version 5.02.

\section{Competing interests}

The authors declare that they have no competing interests.

\section{Authors' contributions}

WC, S-SF and SM conceived and designed the experiments. WC and S-SF performed the experiments. S-SF and WC analyzed the data. RWL and PNS contributed reagents/materials/analysis tools. S-SF, WC and SM wrote the manuscript. All authors read and approved the final manuscript.

\section{Acknowledgements}

SM is the recipient of an Australian National Health and Medical Research Council (NHMRC) Senior Research Fellowship (APP1059167). This project was supported by funding from the Australian NHMRC grant to SM (grant APP1047252).

\section{Author details}

'Emerging Viruses and Inflammation Research Group, Institute for Glycomics, Griffith University, Gold Coast Campus, QLD 4222, Australia. ${ }^{2}$ Trauma and Orthopaedic Research Unit Laboratory, The Medical School, The Australian National University, Garran Rd, Canberra ACT 2601, Australia. ${ }^{3}$ Department of Orthopaedic Surgery, Trauma and Orthopaedic Research Unit, The Canberra Hospital, Canberra ACT 2605, Australia.

Received: 17 July 2014 Accepted: 26 October 2014

Published online: 19 November 2014

\section{References}

1. Suhrbier A, Jaffar-Bandjee MC, Gasque P: Arthritogenic alphaviruses-an overview. Nat Rev Rheumatol 2012, 8:420-429.

2. Colin de Verdière N, Molina J-M: Rheumatic manifestations caused by tropical viruses. Joint Bone Spine 2007, 74:410-413.

3. Harley D, Bossingham D, Purdie DM, Pandeya N, Sleigh AC: Ross River virus disease in tropical Queensland: evolution of rheumatic manifestations in an inception cohort followed for six months. Med J Aust 2002, 177:352-355.
4. Rulli NE, Melton J, Wilmes A, Ewart G, Mahalingam S: The molecular and cellular aspects of arthritis due to alphavirus infections: lesson learned from Ross River virus. Ann N Y Acad Sci 2007, 1102:96-108.

5. Suhrbier A, La Linn M: Clinical and pathologic aspects of arthritis due to Ross River virus and other alphaviruses. Curr Opin Rheumatol 2004, 16:374-379.

6. Schwartz O, Albert ML: Biology and pathogenesis of chikungunya virus. Nat Rev Microbiol 2010, 8:491-500.

7. Chaaitanya IK, Muruganandam N, Sundaram SG, Kawalekar O, Sugunan AP, Manimunda SP, Ghosal SR, Muthumani K, Vijayachari P: Role of proinflammatory cytokines and chemokines in chronic arthropathy in CHIKV infection. Viral Immunol 2011, 24:265-271.

8. Chow A, Her Z, Ong EK, Chen JM, Dimatatac F, Kwek DJ, Barkham T, Yang H, Renia L, Leo YS, Ng LF: Persistent Arthralgia Induced by Chikungunya Virus Infection is Associated with Interleukin-6 and Granulocyte Macrophage Colony-Stimulating Factor. J Infect Dis 2011, 203:149-157.

9. Russell RC: ROSS RIVER VIRUS: Ecology and Distribution. Annu Rev Entomol 2002, 47:1

10. Nakamura I, Takahashi N, Jimi E, Udagawa N, Suda T: Regulation of osteoclast function. Mod Rheumato/ 2012, 22:167-177.

11. Firestein GS: Evolving concepts of rheumatoid arthritis. Nature 2003, 423:356-361.

12. Goldring S: Pathogenesis of bone and cartilage destruction in rheumatoid arthritis. Rheumatology 2003, 42:ii11-ii16.

13. Ainola M, Mandelin J, Liljeström M, Konttinen $Y T$, Salo J: Imbalanced expression of RANKL and osteoprotegerin mRNA in pannus tissue of rheumatoid arthritis. Clin Exp Rheumatol 2008, 26:240-246.

14. Giner M, Rios MJ, Montoya MJ, Vázquez MA, Naji L, Pérez-Cano R: RANKL/ OPG in primary cultures of osteoblasts from post-menopausal women. Differences between osteoporotic hip fractures and osteoarthritis. J Steroid Biochem Mol Biol 2009, 113:46-51.

15. van Tuyl LHD, Voskuyl AE, Boers M, Geusens P, Landewé RBM, Dijkmans BAC, Lems WF: Baseline RANKL:OPG ratio and markers of bone and cartilage degradation predict annual radiological progression over 11 years in rheumatoid arthritis. Ann Rheum Dis 2010, 69:1623-1628.

16. Manimunda SP, Vijayachari P, Uppoor R, Sugunan AP, Singh SS, Rai SK, Sudeep AB, Muruganandam N, Chaitanya IK, Guruprasad DR: Clinical progression of chikungunya fever during acute and chronic arthritic stages and the changes in joint morphology as revealed by imaging. Trans R Soc Trop Med Hyg 2010, 104:392-399.

17. Chen W, Foo SS, Rulli NE, Taylor A, Sheng KC, Herrero L, Herring BL, Lidbury BA, Li RW, Walsh NC, Sims NA, Smith PN, Mahalingam S: Arthritogenic alphaviral infection perturbs osteoblast function and triggers pathologic bone loss. Proc Natl Acad Sci U S A 2014, 111:6040-6045.

18. Woolf AD, Pfleger B: Burden of major musculoskeletal conditions. Bull World Health Organ 2003, 81:646-656.

19. Kapoor M, Martel-Pelletier J, Lajeunesse D, Pelletier JP, Fahmi H: Role of proinflammatory cytokines in the pathophysiology of osteoarthritis. Nat Rev Rheumatol 2011, 7:33-42.

20. Mylonas AD, Brown AM, Carthew TL, McGrath B, Purdie DM, Pandeya N, Vecchio PC, Collins LG, Gardner ID, de Looze FJ, Reymond EJ, Suhrbier A: Natural history of Ross River virus-induced epidemic polyarthritis. Med J Aust 2002, 177:356-360.

21. Barber B, Denholm JT, Spelman D: Ross River virus. Aust Fam Physician 2009, 38:586-589.

22. Pincus T: Clinical evidence for osteoarthritis as an inflammatory disease. Curr Rheumatol Rep 2001, 3:524-534.

23. Takayanagi $\mathrm{H}$ : Osteoimmunology: shared mechanisms and crosstalk between the immune and bone systems. Nat Rev Immunol 2007, 7:292-304

24. Yadav A, Saini V, Arora S: MCP-1: chemoattractant with a role beyond immunity: a review. Clin Chim Acta 2010, 411:1570-1579.

25. Sissoko D, Malvy D, Ezzedine K, Renault P, Moscetti F, Ledrans M, Pierre V: Post-epidemic Chikungunya disease on Reunion Island: course of rheumatic manifestations and associated factors over a 15-month period. PLoS Negl Trop Dis 2009, 3:e389.

26. Kularatne SA, Weerasinghe SC, Gihan C, Wickramasinghe S, Dharmarathne S, Abeyrathna A, Jayalath T: Epidemiology, clinical manifestations, and longterm outcomes of a major outbreak of chikungunya in a hamlet in Sri Lanka, in 2007: a longitudinal cohort study. J Trop Med 2012, 2012:639178.

27. Noret M, Herrero L, Rulli N, Rolph M, Smith PN, Li RW, Roques P, Gras G, Mahalingam S: Interleukin 6, RANKL, and osteoprotegerin expression by 
chikungunya virus-infected human osteoblasts. J Infect Dis 2012, 206:455-457-457-459.

28. Heise MT, Simpson DA, Johnston RE: Sindbis-group alphavirus replication in periosteum and endosteum of long bones in adult mice. J Virol 2000, 74:9294-9299.

29. Couderc T, Chrétien F, Schilte C, Disson O, Brigitte M, Guivel-Benhassine F, Touret Y, Barau G, Cayet N, Schuffenecker I, Desprès P, Arenzana-Seisdedos F, Michault A, Albert ML, Lecuit M: A Mouse Model for Chikungunya: Young Age and Inefficient Type-I Interferon Signaling Are Risk Factors for Severe Disease. PLoS Pathog 2008, 4:e29.

30. Gardner CL, Burke CW, Higgs ST, Klimstra WB, Ryman KD: Interferon-alpha/ beta deficiency greatly exacerbates arthritogenic disease in mice infected with wild-type chikungunya virus but not with the cell cultureadapted live-attenuated 181/25 vaccine candidate. Virology 2012, 425:103-112.

31. Kato H, Takeuchi O, Sato S, Yoneyama M, Yamamoto M, Matsui K, Uematsu S, Jung A, Kawai T, Ishii KJ, Yamaguchi O, Otsu K, Tsujimura T, Koh CS, Reis e Sousa C, Matsuura Y, Fujita T, Akira S: Differential roles of MDA5 and RIG-I helicases in the recognition of RNA viruses. Nature 2006, 441:101-105.

32. Yoneyama M, Kikuchi M, Natsukawa T, Shinobu N, Imaizumi T, Miyagishi M, Taira K, Akira S, Fujita T: The RNA helicase RIG-I has an essential function in double-stranded RNA-induced innate antiviral responses. Nat Immunol 2004, 5:730-737.

33. Boyce BF, Xing L: Functions of RANKL/RANK/OPG in bone modeling and remodeling. Arch Biochem Biophys 2008, 473:139-146.

34. Sims NA, Walsh NC: GP130 cytokines and bone remodelling in health and disease. BMB Rep 2010, 43:513-523.

35. Takemura M, Harada A, Mizuno M, Yano K, Yamada Y: Relationship between osteoprotegerin/osteoclastogenesis inhibitory factor concentration in synovial fluid and disease severity in individuals with osteoarthritis of the knee. Metabolism 2001, 50:1-2.

36. Skoumal M, Kolarz G, Haberhauer G, Woloszczuk W, Hawa G, Klingler A: Osteoprotegerin and the receptor activator of NF-kappa B ligand in the serum and synovial fluid. A comparison of patients with longstanding rheumatoid arthritis and osteoarthritis. Rheumatol Int 2005, 26:63-69.

37. Hoarau J-J, Jaffar Bandjee M-C, Krejbich Trotot P, Das T, Li-Pat-Yuen G, Dassa B, Denizot M, Guichard E, Ribera A, Henni T, Tallet F, Moiton MP, Gauzère BA, Bruniquet S, Jaffar Bandjee Z, Morbidelli P, Martigny G, Jolivet M, Gay F, Grandadam M, Tolou H, Vieillard V, Debré P, Autran B, Gasque P: Persistent chronic inflammation and infection by Chikungunya arthritogenic alphavirus in spite of a robust host immune response. J Immunol 2010, 184:5914-5927.

38. Lidbury BA, Rulli NE, Suhrbier A, Smith PN, McColl SR, Cunningham AL, Tarkowski A, van Rooijen N, Fraser RJ, Mahalingam S: Macrophage-derived proinflammatory factors contribute to the development of arthritis and myositis after infection with an arthrogenic alphavirus. J Infect Dis 2008, 197:1585.

39. Kuhn RJ, Niesters HG, Hong Z, Strauss JH: Infectious RNA transcripts from Ross River virus CDNA clones and the construction and characterization of defined chimeras with Sindbis virus. Virology 1991, 182:430-441.

40. Morrison TE, Whitmore AC, Shabman RS, Lidbury BA, Mahalingam S, Heise MT: Characterization of Ross River Virus Tropism and Virus-Induced Inflammation in a Mouse Model of Viral Arthritis and Myositis. J Virol 2006, 80:737-749.

doi:10.1186/s12985-014-0189-9

Cite this article as: Chen et al:: Osteoblasts from osteoarthritis patients show enhanced susceptibility to Ross River virus infection associated with delayed type I interferon responses. Virology Journal 2014 11:189.

\section{Submit your next manuscript to BioMed Central and take full advantage of:}

- Convenient online submission

- Thorough peer review

- No space constraints or color figure charges

- Immediate publication on acceptance

- Inclusion in PubMed, CAS, Scopus and Google Scholar

- Research which is freely available for redistribution 